\title{
L'emodialisi e la politerapia farmacologica: indagine sull'aderenza ai farmaci in un gruppo di pazienti in emodialisi presso il Centro Dialisi S. Vito
}

\author{
E. Di Mari ${ }^{1}$, G.P. Segoloni ${ }^{2}$, M.Talaia ${ }^{3}$
}

${ }^{1}$ Corso di Laurea in Infermieristica, Facoltà di Medicina e Chirurgia, Università degli Studi di Torino, Torino

${ }^{2}$ Direttore S.C. Nefrologia, Dialisi e Trapianto A.O.U. S. Giovanni Battista di Torino, Torino

${ }^{3}$ Coordinatore infermieristico Centro Dialisi, Ospedale S. Vito, Torino

HAEMODIALYSIS AND DRUG POLITHERAPY: THE COMPLIANCE TO THE THERAPEUTIC PRODUCTS IN A POPULATION OF DIALYSED PATIENTS AT S. Vito CENTER

Abstract. Adherence to treatments represents one of the most successful clinical factor in patients undergoing chronic disease, like CKD. Althought, many factors could decrease adherence, nurses and doctors are called to strengthen the patients partnership in order to improved it.

This study refers to a survey which took place with 56 patients, of a sample of 70, who were undergoing dialysis treatment in San Vito Hospital in Turin. The study helped to enhance the elements which more influence drug adherence, home drug compliance, drug knowledge and expected clinical outcomes. These datas contributed to understand patients' educational needs, areas and topic that doctors and nurses should look for to get better clinical outcomes.

To forget treatment pills had shown to be the most frequent reason for non adherence, followed by unfriendly prescribed time-schedule that hardly matched with ones's life style. The given questionnaire showed that better drug knowledge and limited number of pills decreased the burdening of treatments, thas increasing patients' adherence. On the contrary, clear provision of information from health personals did not show to be relevant key factor. At the end of the study the caring group decided up the implementation of some actions in order to improve patients' outcomes.

Key Words. Adherence, Dialysis, Politherapy, Drugs

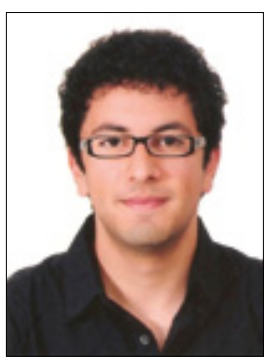

Emanuele Di Mari

\section{Introduzione}

La scarsa aderenza alla terapia rappresenta tutt'oggi una delle barriere più delicate per il raggiungimento del risultato clinico voluto; è infatti noto che circa un paziente su quattro non segue adeguatamente la prescrizione farmacologica indicatagli dal proprio medico (1).

Garantire un'aderenza ottimale diventa complesso nel caso di pazienti con patologie croniche, che prevedono l'assunzione dei farmaci per lunghi periodi di tempo. Il paziente uremico, rientra in questa categoria di perso- ne che per sopravvivere deve attenersi a un regime terapeutico comprendente, oltre alla dialisi, la politerapia farmacologica.

Nonostante il medico si avvalga delle evidenze scientifiche per la prescrizione farmacologica, il destino della terapia e dell'aderenza è nelle mani del paziente.

Ricercare i fattori che determinano la non aderenza significa entrare nell'ottica del paziente, esplorare le sue caratteristiche e adeguare il trattamento alla malattia, alle aspettative del paziente, alle sue risorse, al suo stile di vita e ai suoi valori.

Osterberg e Blaschke (2) riportano i motivi di non assunzione dei farmaci prescritti forniti dai pazienti. Questi, in ordine di frequenza, includono: 
- dimenticanza;

- altre priorità;

- decisione di saltare una dose;

- mancanza di informazioni adeguate;

- fattori emozionali.

Tra questi, l'elemento sul quale il medico e l'infermiere possono agire è sicuramente la mancanza di informazioni adeguate.

Dai risultati di uno studio di Catz et al (3), che hanno indagato sulle strategie utilizzate dai pazienti per favorire l'aderenza alle prescrizioni farmacologiche, risulta che il 94\% del campione chiede informazioni sui farmaci a un medico, un infermiere o a un farmacista.

Linfermiere essendo a più stretto contatto col paziente, può costituire una preziosa fonte di informazioni, chiarimenti e consigli pratici per quanto riguarda la gestione farmacologica domiciliare.

Non sempre però le capacità comunicative e relazionali dell'infermiere sono adeguate alle richieste di informazioni del paziente, che può perdere fiducia in tale figura, compromettendo l'alleanza terapeutica stabilita e l'aderenza alla terapia prescritta.

Risulta quindi opportuno esplorare quanto l'aderenza del paziente all'assunzione dei farmaci a domicilio possa essere influenzata dalle informazioni ricevute, dalla conoscenza della terapia e dal personale sanitario.

L'obiettivo dello studio è quello di individuare la diffusione della scarsa aderenza alla terapia farmacologica in un gruppo di pazienti emodializzati; mettere l'aderenza in relazione al numero di farmaci prescritti, alle conoscenze del paziente sulla terapia assunta e alle informazioni ricevute dal personale sanitario, al fine di ricercare le criticità e gli interventi infermieristici più idonei.

\section{Materiali e Metodi}

\section{Contesto e campione}

Lo studio è stato condotto presso il C.A.L. dell'Ospedale S. Vito di Torino, sede distaccata della S.C. di Nefrologia, Dialisi e Trapianto dell'A.O.U. San Giovanni Battista di Torino.

Dei 70 assistiti seguiti dal Centro dialitico del S. Vito durante il periodo di raccolta dati (9-13 dicembre 2011) sono stati esclusi dallo studio 14 pazienti per aver negato il consenso, per assenza o per mancata comprensione dell'italiano/inglese.

Il campione è rappresentato da 56 pazienti, di cui 54 italiani e 2 stranieri suddivisi in 38 individui maschi e 18 femmine, di età media compresa tra i 66 e i 75 anni.

Si tratta prevalentemente di persone in pensione $(66 \%)$ che vivono da sole $(41 \%)$ o con un convivente $(52 \%)$. Sol- tanto il 9\% risulta disoccupato, mentre il resto mantiene un'attività lavorativa a tempo pieno (12\%), part-time $(9 \%)$ o autonoma (4\%).

L'età dialitica è per il $48 \%$ dei pazienti compresa tra 0 e 5 anni, per il $20 \%$ tra i 6 e i 10 anni mentre supera i 15 anni di dialisi il 18\% del campione.

Il 34\% dei pazienti possiede la licenza media, il 32\% è diplomato, il $20 \%$ ha licenza elementare e solo il $14 \%$ è laureato.

\section{Metodo di raccolta dati}

I dati sono stati raccolti tramite self report, predisponendo un questionario ad hoc, anonimo, costituito da 23 domande sia a risposta chiusa che aperta, suddiviso in 2 parti: una riguardante i dati socio-anagrafici del paziente, l'altra indaga le conoscenze del paziente su ogni singolo farmaco prescritto e sugli effetti della sua mancata assunzione; sull'aderenza al regime terapeutico e i metodi usati a domicilio dal paziente per supportarla e sulla qualità delle informazioni ricevute dal personale sanitario. La somministrazione è avvenuta durante la seduta dialitica mediante intervista guidata, al fine di fornire chiarimenti alle domande proposte e di massimizzare la quantità di dati ottenuti.

Sono state inoltre consultate le cartelle cliniche dei pazienti al fine di confrontare alcuni risultati.

\section{Risultati}

Dai risultati del questionario è emerso che l'intero campione assume la terapia a domicilio, con una media di 7.25 tipi di farmaco diverso per paziente.

Il metodo più utilizzato dagli assistiti per ricordare di assumere i farmaci a domicilio è la memoria (56\%). Il resto dei pazienti si affida al foglio della terapia (16\%) o a un badante che ne gestisce orari e dosi (15\%). Il 7\% è solito suddividere i farmaci in 3 scatolette (mattino, pomeriggio e sera), il $4 \%$ utilizza un'agenda per segnare le assunzioni e il $2 \%$ prepara la sera prima tutti i farmaci del giorno dopo.

E stato chiesto ai pazienti di elencare e spiegare in termini semplici l'azione terapeutica di ogni farmaco prescritto. A coloro che hanno ricordato solo il nome del farmaco ma che non hanno descritto il motivo di assunzione è stata assegnata una risposta negativa $(\mathrm{No})$, mentre a coloro che hanno dimostrato di sapere l'utilizzo di ogni singolo farmaco è stata assegnata una risposta positiva (Si, tutti).

I risultati dimostrano che il $70 \%$ degli intervistati è consapevole di ciò che assume mentre al restante $30 \%$ mancano le conoscenze di uno o più farmaci assunti. Il mo- 
tivo principale di scarsa conoscenza è la dimenticanza, favorita dal numero di farmaci prescritti.

Al fine di identificare la diffusione della non aderenza è stato chiesto ai pazienti se capitasse loro di non assumere la terapia prescritta ed è risultato che il $34 \%$ ha risposto "mai", il 25\% "raramente", il 29\% "qualche volta" e il $12 \%$ "spesso".

I motivi riferiti riguardano prevalentemente la dimenticanza (55\%) dovuta all'elevato numero di farmaci prescritti, mentre il $21 \%$ non riesce a coniugare lo stile di vita e il lavoro con la posologia dei farmaci. Il $12 \%$ non trova utile il farmaco dal quale non ottiene benefici concreti. Per il 5\% del campione la mancata assunzione della terapia prescritta è dovuta agli effetti collaterali.

Un altro $5 \%$ sostiene di stare bene, pertanto i farmaci non determinano il proprio stato di salute.

Il $2 \%$ del campione rifiuta i farmaci per il numero eccessivo.

Le informazioni sulla terapia ricevute dai medici sono completamente chiare per il $64 \%$ del campione, il restante $36 \%$ le ha ritenute poco o non chiare. Il $60 \%$ dei pazienti sostiene che gli infermieri trasmettono informazioni chiare, 1 ' $11 \%$ che queste sono poco o non chiare, mentre il $29 \%$ non chiede mai informazioni sulla terapia agli infermieri, in quanto ritiene che l'argomento sia esclusivamente di competenza medica.

Il $48 \%$ dei pazienti, in caso di dubbi sulla terapia, non chiede mai informazioni al personale sanitario. Nonostante ciò, circa l' $80 \%$ del campione si ritiene soddisfatto delle capacità degli operatori sanitari di trasmettere informazioni relative ai farmaci assunti a domicilio. I pazienti meno soddisfatti vorrebbero più tempo da dedicare all'argomento e una trasmissione delle informazioni più chiara.

\section{Discussione}

Per indagare sulla diffusione della non aderenza sono stati considerati aderenti i pazienti che alla domanda «Le capita di non assumere i farmaci prescritti?» hanno risposto "raramente" e "mai" (59\%) e come meno aderenti i pazienti che hanno risposto "qualche volta" e "spesso" (41\%).

Le tre più frequenti motivazioni di scarsa aderenza fornite dai pazienti dello studio (dimenticanza, terapia non in linea con lo stile di vita e non lo trovo utile) sono risultate identiche sia per i pazienti considerati aderenti sia per i meno aderenti e sono sovrapponibili a quelle riportate da Osterberg e Blaschke.

Confrontando la media di farmaci prescritti risultante dalle cartelle cliniche (11.09) con quella riferita dai pazienti (7.25) si è notato che la memoria, strategia più usata dal campione, non risulta efficace come metodo di supporto all'aderenza.

Inoltre il numero (non dosi) di farmaci assunti quotidianamente dagli aderenti è in media di 6.9, mentre quello dei meno aderenti è di 7.5. La lieve differenza è resa più consistente dal quantitativo di dosi giornaliere prescritte per ciascun farmaco, che aumenta il numero di assunzioni quotidiane. Ciò dimostra che un maggior numero di farmaci influisce sull'aderenza.

I pazienti che sanno a cosa servono i farmaci prescritti (70\%) corrispondono a quelli che conoscono le conseguenze della loro mancata assunzione. A parità di conoscenza di farmaci assunti, il $62 \%$ dei pazienti è risultato aderente. Ciò dimostra che un paziente informato sull'azione terapeutica di ciò che gli è prescritto lo incentiva ad essere più aderente.

La maggior chiarezza delle informazioni fornite dai medici e dagli infermieri non contribuisce in modo significativo a migliorare l'aderenza alla terapia. Infatti i pazienti che hanno ricevuto informazioni chiare, poco o non chiare sono risultati per la maggior parte aderenti. Dai dati ottenuti, è emersa la presenza di una barriera comunicativa tra pazienti e infermieri. Infatti il $29 \%$ dei pazienti non chiede mai informazioni sulla terapia agli infermieri. Questi, non conoscendo il bisogno informativo degli assistiti, non dedicano abbastanza tempo all'argomento e non dimostrano ai pazienti di avere le conoscenze sui farmaci da loro assunti. A volte tale barriera comunicativa è rafforzata dalla scarsa conoscenza degli infermieri sui nuovi farmaci introdotti, che li portano ad evitare l'argomento.

\section{Conclusioni}

Alle criticità emerse dallo studio, sono stati proposti i seguenti interventi:

- Gli operatori sanitari potrebbero concentrare l'attenzione sulle metodologie usate dai pazienti per far fronte al problema della non aderenza, rivalutandone assieme al paziente l'efficacia, fornendo maggior supporto o proponendo di cambiare il metodo utilizzato.

- In merito alle conoscenze sui farmaci assunti, per poter ridurre il numero dei pazienti che nonostante ne sia in possesso risulta meno aderente, l'infermiere potrebbe periodicamente provvedere a verificare se il paziente sia informato sui farmaci che gli sono stati prescritti, sul motivo di prescrizione e sulla loro azione, per poter chiarire gli eventuali dubbi e migliorare la consapevolezza del paziente sulla terapia assunta. 
- Organizzare incontri informativi tra medici e infermieri rispetto all'introduzione di nuovi farmaci, in modo da avere le stesse conoscenze e far fronte alla richiesta di chiarimenti al paziente.

- Dedicare più tempo da parte degli infermieri all'argomento farmaci, le cui conoscenze, come dimostrato aumentano l'aderenza, nonché la fiducia del paziente nei suoi confronti, incentivandolo a chiedere in caso di dubbi e aumentando così l'aderenza.

\section{Riassunto}

L'aderenza alla terapia rappresenta uno dei fattori principali di successo clinico soprattutto per i pazienti con patologie croniche come quelli con I.R.C. Spesso però, sono numerosi i fattori che la compromettono e il medico e l'infermiere sono chiamati ad intervenire per rafforzare l'alleanza terapeutica con il paziente al fine di migliorare l'aderenza.

Lindagine condotta in questo studio su 56 dei 70 pazienti emodializzanti nell'ospedale S. Vito di Torino, ha permesso di individuare quali elementi influenzano la loro aderenza alla terapia farmacologica domiciliare, soprattutto nell'ambito delle conoscenze sui farmaci assunti e del supporto informativo fornito dai medici e gli infermieri del Centro Dialisi.

La dimenticanza nell'assumere la terapia si è rivelata tra i fattori principali di non aderenza, seguita dalla posologia delle prescrizioni farmacologiche poco coniugabili con lo stile di vita del paziente. I risultati del questionario somministrato agli assistiti, hanno dimostrato che la conoscenza da parte del paziente sulla terapia assunta e un minor numero di farmaci prescritti, influenzano positivamente la sua aderenza. Al contrario, la chiarezza delle informazioni ricevute dal personale sanitario non è risultata significativa in questo ambito.

Al termine dello studio sono stati individuati alcuni interventi atti ad affrontare le criticità emerse dall'indagine.

Parole Chiave: Aderenza, Dialisi, Politerapia, Farmaci

\author{
Indirizzo degli Autori: \\ Dr. Emanuele Di Mari \\ Via Asiago 72 \\ 96019 Rosolini (SR) \\ emanuele.dimari@gmail.com
}

\section{Bibliografia}

1. Di Matteo MR. Variations in patients' adherence to medical recommendations: a quantitative review of 50 years of research. Med Care 2004; 42: 200-09.

2. Osterberg L, Blaschke T. Adherence to Medication. N Engl J Med 2005; 353: 487-97.

3. Catz SL, Kelly JA, Bogart LM, Benotsch EG, McAuliffe TL. Patterns, correlates, and barriers to medication adherence among persons prescribed new treatments for HIV disease. Health Psychology 2000; 19: 124-33. 